\title{
EXPRESSION OF BASAL AND LUMINAL CYTOKERATINS IN BREAST CANCER AND THEIR CORRELATION WITH CLINICOPATHOLOGICAL PROGNOSTIC VARIABLES
}

\author{
FERESHTEH MOHAMMADIZADEH, AZAR NAIMI, PARVIN RAJABI, HAMIDREZA GHASEMIBASIR, \\ AMIN EFTEKHARI
}

\section{ABSTRACT}

BACKGROUND: Normal breast ducts contain at least 3 types of epithelial cells: luminal (glandular) cells, basal/myoepithelial cells and stem cells. Myoepithelial and luminal epithelia can be distinguished by their different cytokeratin expression patterns. The aim of this study is to evaluate the expression of some prognostic biomarkers (ER, PR and HER2), as well as histological grading and lymph node status in cytokeratin-based groups of breast cancer. OBJ ECTIVE: To evaluate the correlation between expression of basal and luminal markers and hormonal receptors, HER2/neu, age, grade and lymph node status in breast-invasive ductal carcinoma. MATERIALS AND METHODS: Sixtyseven formalin-fixed and paraffin-embedded breast cancer specimens (of invasive ductal carcinoma, 'NOS' type) which had already been studied for ER, PR and HER2/neu were selected. Data concerning age, tumor grade and lymph node status were also obtained from archives. Expression of basal (CK5/6) and luminal (CK7) cytokeratins was detected by immunohistochemistry. Stained sections were classified according to the intensity of staining and the percentage of stained cells. RESULTS: We categorized the cases into 3 distinct phenotype groups: pure luminal, basal phenotype and null. Pure basal, mixed basal and luminal groups were classified as expressing a basal phenotype. There was a significant difference in the ER and/or PR expression between those 3 groups and a significant association between ER and/or PR negativity and basal phenotype expression. There was no significant difference in HER2/neu expression, age of the patients, tumor grade and lymph node status between the 3 cytokeratin-based groups and no significant association between lymph node status and basal phenotype expression. CONCLUSION: We found that to gain a real association between basal phenotype and prognostic markers, we should use a cocktail or a panel of different biomarkers to correctly determine basal-like phenotype of breast cancers. This approach guarantees more concordance with gene expression-based studies.

Key words: Basal, breast cancer, cytokeratin 5/6, immunohistochemistry, luminal DOI: $10.4103 / 0019-5359.50764$

Department of Pathology, Isfahan University of Medical

Sciences, Alzahra Hospital, Isfahan, Iran

Correspondence:

Dr. Azar Naimi,

Department of Pathology, Isfahan University of Medical

Sciences, Alzahra Hospital, Isfahan, Iran.

E-mail: azarnaimi@yahoo.com

\section{INTRODUCTION}

Breast cancer is a major concern and one of the leading causes of cancer-related death worldwide. 
Variations in transcriptional programs account for much of the biological diversity of human cells and tumors. ${ }^{[1]}$ Defining molecular abnormalities in breast cancer is an important strategy for assessing prognosis and treatment. ${ }^{[2]}$

Most investigators have addressed breast carcinoma precursors by analyzing expression of cytokeratin-differentiation markers, since their expression is thought to remain stable throughout carcinogenesis. ${ }^{[3]}$

Prognostic biomarkers for breast cancer include estrogen receptor (ER) and progesterone receptor (PR) overexpression; markers of oncogene overexpression, such as human epidermal growth factor receptor-2 (HER2/neu); indicators of apoptotic imbalance, including overexpression of bcl-2; markers of disordered cell signaling, such as c-myc overexpression; loss of differentiation markers, such as transforming growth factor- $\beta$ II receptor; and alterations of angiogenesis proteins, such as vascular endothelial growth factor (VEGF) overexpression. ${ }^{[2]}$

The most important prognostic factors in current use are clinical features such as lymph node status, tumor size and tumor grade. ${ }^{[4]}$

Determination of estrogen receptor (ER) status in invasive carcinoma prior to therapeutic interventions has become a standard practice for management of breast cancer. Betterdifferentiated tumors are likely to be ERpositive, and these ER-positive tumors have a relatively better prognosis. Conversely, ERnegative tumors are more likely to be of higher histological grade, and the patients more likely to have a decrease in survival depending on age and lymph node status. ${ }^{[5]}$

Normal breast ducts contain at least 3 types of epithelial cells: luminal (glandular) cells, basal/ myoepithelial cells and stem cells. Myoepithelial and luminal epithelia can be distinguished by their different cytokeratin expression patterns. Myoepithelial cells typically express cytokeratin $5 / 6$ and cytokeratin 17 , while luminal cells typically express cytokeratins 8 and $18 .^{[5]}$

A small fraction of breast cancers express CK5 together with its major partners CK14 and CK17. ${ }^{[6]}$

Basal-like tumors express many of the genes characteristic of breast basal epithelial cells. ${ }^{[7]}$ The most typical feature of basal-like breast cancer is the lack of expression of ER and genes usually co-expressed with ER. ${ }^{[8]}$

The precise prevalence and clinicopathological characteristics of basal and luminal CKexpressing and co-expressing tumors remain unclear. ${ }^{[3]}$ In particular, the presence of any association between HER2/neu amplification and either basal or luminal phenotypes is currently unknown. The results of some studies have suggested that tumors with basal phenotype express HER2 protein, ${ }^{[9]}$ while others have shown lack of HER2 expression in basal-like tumors. ${ }^{[10]}$

In a study by Rijin et al., $16 \%$ of tumors were positive for cytokeratin $5 / 6$ or cytokeratin 17 , and basal cytokeratin expression was associated with a poor prognosis. ${ }^{[4]}$ In the study by Abd El-Rehim, approximately $18 \%$ of invasive breast cancers had basal cytokeratin 
immunoreactivity; and again, these tumors showed a poor prognosis. ${ }^{[11]}$

The breast cancer subtypes have been extensively characterized by gene expression analysis using DNA microarrays. While this remains the gold standard, it is not currently feasible for large-scale clinical applications or retrospective studies on formalin-fixed and paraffin-embedded samples. In these situations, the immunohistochemical staining profile $(\mathrm{IHC})$ can be a useful surrogate of gene expression analysis, ${ }^{[12]}$ although the staining pattern of cytokeratins $5 / 6$ and 17 can be highly variable ${ }^{[4]}$ and basal cytokeratins are not expressed in all tumors classified as basal-like by gene microarray analysis. This emphasizes a need for identifying other markers of basallike subtypes. ${ }^{[1]}$

Some studies have employed basal/ myoepithelial cytokeratins and other markers to identify a subset of ER-negative breast carcinomas that are associated with poor prognosis, further supporting the idea that a basal-like phenotype exists. ${ }^{[10,13,14]}$ However, some features point towards the heterogeneous nature of an ER-negative subgroup of invasive breast cancers. ${ }^{[15,16]}$

Considering this heterogeneity, in this study, we first examined the immunohistochemical pattern of CK5/6 (basal) and CK7 (luminal) expression in sections prepared from formalinfixed and paraffin-embedded tissue samples and then evaluated the expression of some prognostic biomarkers (ER, PR, HER2), as well as histological grading and lymph node status in cytokeratin-based groups of breast cancer.
The aim of this study was to determine if IHCbased basal and luminal grouping of breast cancer could reveal the correlation between cytokeratin expression and clinicopathological markers or if it could estimate indirect outcome in each patient.

\section{MATERIALS AND METHODS}

\section{Clinicopathological review}

Seventy formalin-fixed and paraffin-embedded breast carcinoma samples were selected from the pathology archives of Alzahra Hospital (Isfahan, Iran) from the patients who had undergone surgery between 2004 and 2007 (retrospective analysis).

Inclusion criterion was 'archived primary breast tumors' (T-stage 1-3 invasive ductal carcinoma of 'NOS' type) assessed by immunohistochemistry for the expression of ER, PR and HER2/neu at the time of diagnosis. The histological parameters of all cases were reviewed by 2 pathologists (M.Z. and N), and the histological grade was determined for each case according to Nottingham Modification of the Bloom and Richardson Grading System. The grade is determined by adding up the scores for tubule formation $(>75 \%, 10 \%$ to $75 \%,<10 \%$ ), nuclear pleomorphism (minimal, moderate, marked) and mitotic count ( 0 to 5 , 6 to $10,>11$ per 10 high-power fields); each is given a score of 1,2 or 3 points. This produces a total score between 3 and 9 . Final grading is as follows: 3 to 5 points $=$ grade I; 6 to 7 points $=$ grade II; and 8 to 9 points $=$ grade $\mathrm{III}$.

Slides of all patients' axillary lymph nodes were reviewed. For prognostic purposes, lymph node status was scored according to TNM staging system as follows: 
0 : negative nodes; 1 : one to three positive nodes; 2: four to nine positive nodes; 3 : ten or more positive nodes.

Patients' age data was available, and the patients were categorized in 3 groups, using this data:

1: patients aged $\leq 40$ years; 2 : patients aged between 41 and 60 years; 3 : patients aged $>60$ years.

\section{Immunohistochemistry and scoring:}

The method of immunohistochemical staining for ER, PR and HER2/neu was selected according to standard techniques: 4-micron sections were prepared from the blocks, deparaffinized in xylene, rehydrated in a series of graded alcohols, and placed in a Tris buffer bath [pH, 7.6].

Endogenous peroxidase activity was quenched using $3 \%$ hydrogen peroxide. Slides were rinsed with de-ionized water and placed in a Tris buffer bath.

After incubation, sections were stained with primary antibodies. Appropriate controls were used to ensure uniformity of results.

\section{The antibodies used were as follows:}

1. ER: mouse monoclonal antihuman ER (DAKO, D5, code number: M7047)

2. PR: mouse monoclonal antihuman $P R$ (DAKO, clone PgR 636, N1630)

3. HER2/neu: polyclonal rabbit antihuman c-erbB-2 oncoprotein (DAKO)

All immunostained sections were reviewed and scored by the authors. Expression of ER and
PR was scored between 0 and 2 as follows: 0 (negative): less than $5 \%$ of nuclei staining; 1 (borderline): $5 \%-19 \%$ of nuclei staining; 2 (positive): more than $20 \%$ of nuclei staining.

Expression of HER2/neu was scored 0 to 3 as follows: 0 (negative): no membranous staining identified; 1 (negative): faint staining involving a portion of the circumference of the cytoplasmic membrane of at least $10 \%$ of neoplastic cells; 2 (positive): weak but definitive staining of the membrane over $100 \%$ of the cytoplasmic circumference in at least $10 \%$ of neoplastic cells; 3 (positive): strong positive staining of the membrane over $100 \%$ of the cytoplasmic circumference in at least $10 \%$ of neoplastic cells.

Sections of length $4 \mu \mathrm{m}$ were prepared from formalin-fixed and paraffin-embedded tissue samples for Ccytokeratin 5/6 and cytokeratin 7 immunohistochemical staining using Envision method. CK5/6 expression was detected by mouse antihuman antibody clone D5/ 16B4, Dakocytomation, Denmark; and CK7 expression was detected by mouse antihuman antibody clone OV-TL 12/30, Dakocytomation, Denmark. Sections were placed on poly-Ilysine slides and dried in an oven at $60^{\circ} \mathrm{C}$ for 60 minutes. Thereafter, sections were deparaffinized, rehydrated and rinsed in tap water before antigen retrieval by proteins-K. After incubation with $3 \%$ hydrogen peroxide, the sections were incubated with monoclonal antibody. Diaminobenzidine was used as the chromogen.

Skin and normal breast tissue were used as positive controls. For negative controls, we omitted the primary antibodies. Cases in which 
no tissue was present on the sections; or in which the material sampled consisted only of fat, fibrosis, normal mammary glands; or cases of in situ carcinoma were omitted from further analysis. Finally, 67 samples fulfilled the criteria to be included in the study. The sections stained for CK5/6 and CK7 were scored by the authors as follows: tumors were classified based on the intensity of staining and the percentage of cells showing cytokeratin expression.

Percentage of cells showing CK5/6 and CK7 expression

$0: 0 \%$

1: up to $10 \%$

$2:>10 \%$

Scoring of staining intensity (membranous and cytoplasmic)

0 : no staining of the cells

1 : weak staining of the cells

2: moderate/strong staining of the cells

These were then multiplied and combined into a final score as follows:

0 (negative): $\leq 1$

1 (positive): $\geq 2$

Patients' follow-up was not documented, and disease-free and overall survival data were not available. Instead, we used prognostic biomarkers, tumor grade and lymph node status for prognostic purposes.

\section{Statistical analysis}

Statistical analysis was performed using SPSS 12.0 statistical software. Correlation between different markers was carried out using Pearson's correlation coefficient.
A $P$-value $<0.05$ was considered significant.

\section{RESULTS}

In normal breast, CK5/6 stains the basal layer of breast ductal epithelium, while CK7 stains luminal cells.

In our study, the frequencies of CK7 and CK5/6 expression in final scoring groups were as follows: 62 (92.5\%) out of 67 cases fell into positive group for CK7, and 5 (7.5\%) out of 67 fell into negative group for this marker.

In case of CK5/6, 32 (47.8\%) out of 67 cases showed positivity for this marker, and 35 $(52.2 \%)$ out of 67 showed negativity [Figure 1].

We categorized the cases into 3 distinct phenotype groups: pure luminal (expression of CK7 only), mixed basal and luminal or basal (expression of CK7 and/or CK5/6, basal phenotype), and null (no expression of CK7 or CK5/6). According to the above classification, the frequencies in the 3 groups were as in Table 1.

Our pure basal group had 1 case only. This group could not be considered a representative sample in any statistical analysis, so we removed this group from our analysis and just considered the mixed basal group.

Table 2 shows the frequencies of ER, PR

Table 1: Frequencies of the cases in distinct phenotypic groups

\begin{tabular}{lc}
\hline Phenotype categories & Frequency \% \\
\hline Pure luminal & $31(46.3)$ \\
Null & $4(6)$ \\
Basal & $32(47.8)$ \\
\hline
\end{tabular}




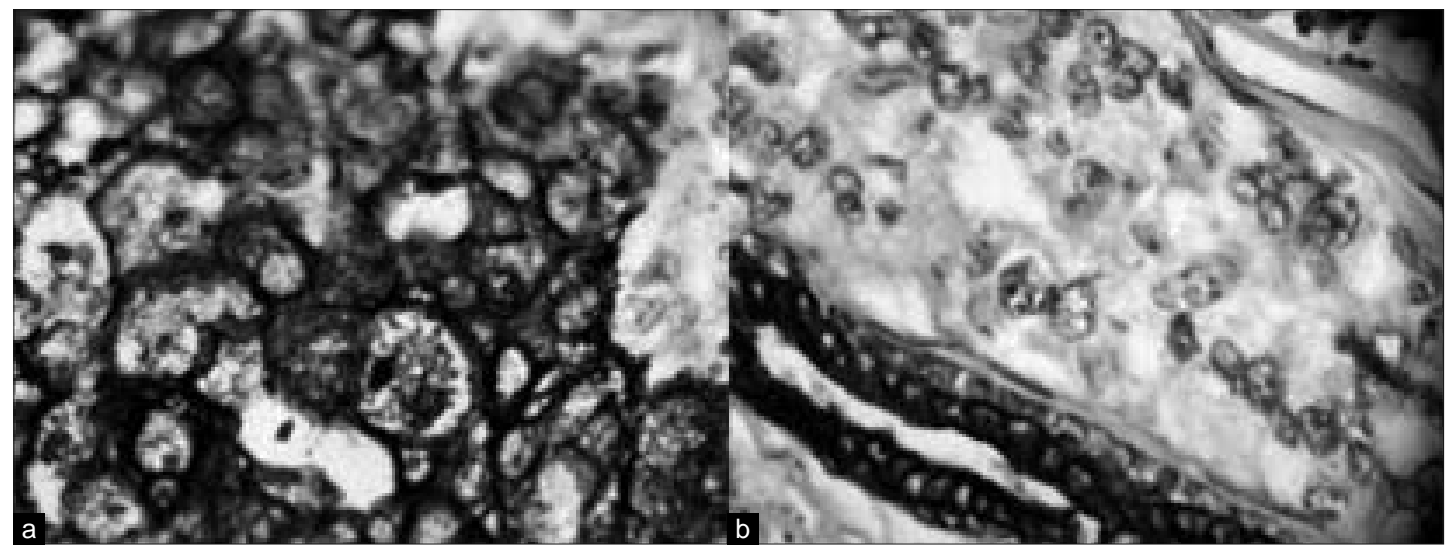

Figure 1: Cytokeratin CK7 staining (strongly positive) (a) and no staining with internal control (b) (Immunohistochemical staining, $\times 400)$

Table 2: Frequencies of expression of biomarkers, tumor grade, age groups and lymph node status of the cases

\begin{tabular}{lccccc}
\hline $9(13.4)$ & I & Grade & $27(40.3)$ & Negative & \\
$26(38.8)$ & II & & $30(44.8)$ & Borderline & ER \\
$32(47.8)$ & III & & $10(14.9)$ & Positive & PR \\
$11(16.4)$ & $<40$ & Age groups & $35(52.2)$ & Negative & \\
$39(58.2)$ & 40 to 60 & & $23(34.3)$ & Borderline & \\
$17(25.4)$ & $>60$ & & $9(13.4)$ & Positive & \\
$18(26.9)$ & Negative & Lymph node status & $27(40.2)$ & Negative(0) & HER2/neu \\
$26(38.8)$ & 1 to 3 & & $18(26.8)$ & Negative(1+) & \\
$19(28.4)$ & 4 to 9 & & $8(11.9)$ & Positive(2+) & \\
$4(6)$ & $\geq 10$ & & $14(20.8)$ & Positive(3+) & \\
\hline
\end{tabular}

Figures in parentheses are in percentage

and HER2/neu expression, as well as tumor grade, lymph node status and age groups of the cases.

Immunohistochemical findings

There was a significant association between ER negativity and basal phenotype expression $(P=0.003)$.

There was a significant association between PR negativity and basal phenotype expression $(P=0.008)$.

There was no significant association between HER2/neu expression and basal phenotype expression.

These results have been summarized in Table 3.
Clinicopathological findings

There was no significant association between age groups and basal phenotype expression $(P=0.192)$.

There was no significant association between tumor grade and basal phenotype expression $(P=0.177)$.

There was no significant association between lymph node status and basal phenotype expression ( $P=0.683)$.

We also did not find any significant difference in the lymph node status, in the form of nodenegative and node-positive, in the basal phenotype group $(P=0.824)$.

These results have been summarized in Table 4. 
Table 3: Frequencies of biomarkers expression in cytokeratin expression-based groups

\begin{tabular}{lcccc}
\hline Basal (mixed) & Null & Pure luminal & Intensity & Biomarker \\
\hline $19(59.4)$ & $1(25)$ & $7(22.6)$ & Negative & ER \\
$12(37.5)$ & $0(0)$ & $18(58.1)$ & Borderline & \\
$1(3.1)$ & $3(75)$ & $6(19.4)$ & Positive & PR \\
$23(71.9)$ & $2(50)$ & $10(32.3)$ & Negative & \\
$6(18.8)$ & $1(25)$ & $16(51.6)$ & Borderline & \\
$3(9.4)$ & $1(25)$ & $5(16.1)$ & Positive & \\
$10(31.2)$ & $2(50)$ & $15(48.3)$ & Negative (0) & HER2/neu \\
$10(31.2)$ & $1(25)$ & $7(22.5)$ & Negative (1+) & \\
$3(9.3)$ & $0(0)$ & $5(16.1)$ & Positive $(2+)$ & \\
$9(28.1)$ & $1(25)$ & $4(12.9)$ & Positive $(3+)$ & \\
\hline
\end{tabular}

Figures in parentheses indicate percentages

Table 4: Frequencies of prevalence in age groups, of lymph node status and tumor grades in cytokeratin expression-based groups

\begin{tabular}{lcccc}
\hline Basal (mixed) & Null & Pure luminal & & \\
\hline $7(21.9)$ & $0(0)$ & $4(12.9)$ & 40 & Age \\
$15(46.9)$ & $2(50)$ & $22(71)$ & 40 to 60 & \\
$10(31.3)$ & $2(50)$ & $5(16.1)$ & $>60$ & Grade \\
$6(18.8)$ & $0(0)$ & $3(9.7)$ & I & \\
$9(21.8)$ & $1(25)$ & $16(51.6)$ & II & \\
$17(53.1)$ & $3(75)$ & $12(38.7)$ & III & Lymph node status \\
$9(28.1)$ & $1(25)$ & $8(25.8)$ & Negative & \\
$14(43.8)$ & $1(25)$ & $11(35.5)$ & 1 to 3 & 4 to 9 \\
$8(25)$ & & $1(25)$ & $10(32.3)$ & \\
$1(3.1)$ & $1(25)$ & $2(6.5)$ & $>10$ & \\
\hline
\end{tabular}

Figures in parentheses indicate percentages

\section{DISCUSSION}

We investigated the expression of some biomarkers; and clinicopathological parameters such as age, tumor grade and lymph node status in breast cancers which had been categorized according to the pattern of cytokeratins 5/6 and 7 expression. So, we placed the cases in 3 groups, one of which was 'with mixed basal and/or luminal markers expression.'

The coexpression of basal/myoepithelial and luminal cytokeratins raises the possibility that these tumors may arise from stem cells that subsequently undergo variable degrees of basal and luminal differentiation. ${ }^{[1]}$

In this study, we have shown that hormonal receptor negativity is more frequent in basal groups of breast cancer.

No association was found between HER2/ neu expression, age of patients, grade of tumors or lymph node status on one hand and cytokeratin-based groups on the other.

The role of hormone receptors as a prognostic and therapeutic tool is widely accepted, and estrogen receptor has proven to be a successful target for all ER-positive breast

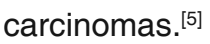

A significant amount of data exists concerning the histological characteristics of ER-negative tumors and the immunohistochemical patterns of expression of nonluminal cell markers (like SMA, p63 and vimentin) in breast carcinomas. The precise relationship between these features and basal-like tumors, however, is 
not known because the only reliable method to identify basal-like tumors is by microarray analysis. ${ }^{[1]}$

While these studies may be more helpful in delineating the basal phenotype as compared to standard immunohistochemical methods, they are not routinely used in clinical practice. The advantage of immunohistochemical method is that it uses markers that are readily available in most pathology departments and is therefore directly translatable into routine clinical management and can be applied to archival specimens for which long-term followup information is already available. ${ }^{[12]}$

Studies have shown basal cytokeratin expression in a subset of invasive breast carcinomas, ${ }^{[11]}$ and it is presumed that most of these tumors are basal-like tumors. However, these studies did not formally demonstrate this relationship, and other classic myoepithelial markers were not typically examined in these studies. ${ }^{[1]}$

Basal-like breast cancer has been associated with poor prognosis in several immunohistochemical[11,17-20] and gene expression microarray-based studies. ${ }^{[20]}$

Nevertheless, there are conflicting results about the independent significance of the basal phenotype. ${ }^{[10,11,18,21,22]}$ Adjuvant chemotherapy could be recognized as one possible confounding factor, because it has been postulated that basal-like and nonbasal tumors would respond differently to chemotherapy. ${ }^{[23]}$

Expression of basal cytokeratins often shows a high degree of intratumoral heterogeneity, ${ }^{[19]}$ which is likely to explain the differences observed between tissue microarrays and entire tissue sections. However, even when performed on entire tumor sections, CK5/14 IHC may not recognize all of the basal-like breast cancers as defined by gene expression profiles. ${ }^{[8]}$ These discrepancies could be because of the differences between findings of gene expression-based and IHC-based studies.

Studies on basal-like breast cancers are likely to be influenced by the ER status, which is a central factor determining both prognosis and gene expression pattern. ${ }^{[7]}$

In our study, there was a significant difference in ER and/or PR expression between basal and luminal groups. This finding shows that combined negativity of ER and PR will improve their correlation with basal phenotype.

Abd El-Rehim et al. classified tumors expressing a basal phenotype (the combined luminal and basal and the pure basal) in a poor prognostic subgroup, typically ER-negative in most cases. ${ }^{[11]}$

Many, but not all, basal-like tumors show expression of $\mathrm{CK} 5 / 6 .{ }^{[10,11]}$ This finding indicates a need for additional immunohistochemical markers to identify all basal-like tumors. Using a limited number of immunohistochemical markers cannot identify all of the real basal-like breast cancers; and therefore, it is insufficient for estimating the outcome of, and response to, therapeutic modalities in basal-like tumors. Antibody cocktails have become increasingly popular in immunohistochemical staining of diagnostic tumor markers. ${ }^{[3]}$ Reliance on the lack of staining for ER and HER2/neu alone 
for identifying basal-like breast cancers carries the risk of mis-assignment based on technical failures and/or biological heterogeneity. ${ }^{[10]}$ The use of a single marker (CK5/6), while successful in identifying a subset of patients with poor outcome, misses approximately half of the basal-like tumors. ${ }^{[10]}$

However, a relatively low staining intensity with some basal markers was found in many cases, which suggests that these tumors might have been scored basal-negative with less sensitive immunohistochemical methods. ${ }^{[3]}$ So weak intensity in IHC staining should be scored as positive. (We did so in our study.)

We did not use CK17 in the immunohistochemical determination of basal cytokeratin expression because some studies had shown previously that only very few tumors show CK17 expression in the absence of CK5 and/or CK14. ${ }^{[3]}$

Our results showed no statistically significant association between $\mathrm{CK} 5 / 6$ expression and HER2/neu overexpression. Published data on HER2/neu and basal phenotype are limited and somewhat conflicting. The results of microarray studies have suggested that basal phenotype breast cancer is HER2/neu nonamplified. ${ }^{[24]}$ In contrast, Birmbaum et al. have reported that basal phenotype breast cancer is associated with HER2 amplification. ${ }^{[25]}$

This study demonstrated that there is overlap in the clinicopathological features such as grade, age and lymph node status of basal and/or luminal breast cancers. It is concluded that immunohistochemically, basal cytokeratin-positive tumors almost always belong to the basal-like gene expression profile, but this cluster also includes some basal cytokeratin-negative tumors. ${ }^{[8]}$ Neither an immunohistochemical nor a microarray-based classification of breast cancers into a basal or nonbasal group is currently considered justified in clinic, because directly predictive or prognostic implications are lacking. ${ }^{[8]}$

In our study, there wasn't any significant difference in lymph node status between cytokeratin-based groups. A recent study has proved lymph node involvement as a less reliable predictor of prognosis in triple-negative phenotype (ER-, PR- and HER2/neu-negative group). ${ }^{[26]}$ In this study, a poor survival was not seen in lymph node-negative basal-like breast cancers, while survival was very poor in lymph node-positive cases. ${ }^{[26]}$ However, other studies have found that node-negative basal-like breast cancer also carries a poor prognosis. ${ }^{[2]]}$

Variable clinical outcome of patients with breast cancer has stimulated the search for powerful prognostic markers which can predict tumor behavior. According to this study,

ER and/or PR negativity in association with a panel of IHC markers may be useful for defining the basal phenotype in breast cancer patients.

\section{CONCLUSION}

In this study, we showed that evaluation of ER and/or PR markers in invasive ductal carcinoma of breast is the basis of determining basal phenotype by cytokeratin 5/6 immunostaining . For gaining a real association, however, we should use a cocktail or a panel of different biomarkers (such as CK14, CK17, SMA and P63) to correctly determine a basal-like 
phenotype of breast cancer, showing more concordance with gene expression-based studies.

Validity of the prognostic significance of basal group breast cancer according to immunohistochemical markers needs a prospective study on a much larger population of patients.

\section{REFERENCES}

1. Livasy CA, Karaca G, Nanda R, Tretiakova MS, Olopade OI, Moore DT, et al. Phenotypic evaluation of the basal-like subtype of invasive breast carcinoma. Mod Pathol 2006;19:264- 71.

2. Fabian CJ, Kimler BF, Elledge RM, Grizzle WE, Beenken SW, Ward JH. Models for early chemoprevention trials in breast cancer. Hematol Oncol Clin North Am 1998;12:993-1017.

3. Laakso M, Loman N, Borg A, Isola J. Cytokeratin 5/14-positive breast cancer: True basal phenotype confined to BRCA1 tumors. Mod Pathol 2005;18:1321-8.

4. van de Rijn M, Perou CM, Tibshirani R, Haas P, Kallioniemi O, Kononen $\mathrm{J}$, et al. Expression of cytokeratins 17 and 5 identifies a group of breast carcinomas with poor clinical outcome. Am J Pathol 2002;161:1991-6.

5. Putti TC, El-Rehim DM, Rakha EA, Paish CE, Lee $A H$, Pinder SE, et al. 5- Estrogen receptornegative breast carcinomas: A review of morphology and immunophenotypical analysis. Mod Pathol 2005;18:26-35.

6. Wetzels $\mathrm{RH}$, Kuijpers $\mathrm{HJ}$, Lane EB, Leigh IM, Troyanovsky SM, Holland R, et al. Basal cellspecific and hyperproliferation-related keratins in human breast cancer. Am J Pathol 1991;138: 751-63.

7. Perou CM, Sørlie T, Eisen MB, van de Rijn M, Jeffrey SS, Rees CA, et al. Molecular portraits of human breast tumours. Nature 2000;406:747-52.
8. Jumppanen M, Gruvberger-Saal S, Kauraniemi $P$, Tanner M, Bendahl PO, Lundin M, et al. Basal-like phenotype is not associated with patient survival in estrogen-receptor-negative breast cancers. Breast Cancer Res 2007;9:R16.

9. Korsching E, Packeisen J, Agelopoulos K, Eisenacher M, Voss R, Isola J, et al. Cytogenetic alterations and cytokeratin expression patterns in breast cancer: Integrating a new model of breast differentiation into cytogenetic pathways of breast carcinogenesis. Lab Invest 2002;82:1525-33.

10. Nielsen TO, Hsu FD, Jensen K, Cheang M, Karaca $\mathrm{G}, \mathrm{Hu} \mathrm{Z}$, et al. Immunohistochemical and clinical characterization of the basal-like subtype of invasive breast carcinoma. Clin Cancer Res 2004;10: 5367-74.

11. Abd El-Rehim DM, Pinder SE, Paish CE, Bell J, Blamey RW, Robertson JF, et al. Expression of luminal and basal cytokeratins in human breast carcinoma. J Pathol 2004;203:661-71.

12. Tischkowitz M, Brunet JS, Bégin LR, Huntsman DG, Cheang MC, Akslen LA, et al. Use of immunohistochemical markers can refine prognosis in triple negative breast cancer. BMC Cancer 2007;7:134.

13. Kesse-Adu R, Shousha S. Myoepithelial markers are expressed in at least $29 \%$ of oestrogen receptor negative invasive breast carcinoma. Mod Pathol 2004;17:646-52.

14. Tsuda H, Takarabe T, Hasegawa F, Fukutomi $\mathrm{T}$, Hirohashi S. Large, central acellular zones indicating myoepithelial tumor differentiation in high-grade invasive ductal carcinomas as markers of predisposition to lung and brain metastases. Am J Surg Pathol 2000;24:197-202.

15. Trendell-Smith NJ, Peston D, Shousha S. Adenoid cystic carcinoma of the breast: A tumour commonly devoid of oestrogen receptors and related proteins. Histopathology 1999;35:241-8.

16. Rosen PP, Cranor ML. Secretory carcinoma of the breast. Arch Pathol Lab Med 1991;115:141-4.

17. Banerjee S, Reis-Filho JS, Ashley S, Steele D, Ashworth A, et al. Basal-like breast carcinomas: 
Clinical outcome and response to chemotherapy. J Clin Pathol 2006;59:729-35.

18. Potemski P, Kusinska R, Watala C, Pluciennik E, Bednarek AK, Kordek R. Prognostic relevance of basal cytokeratin expression in operable breast cancer. Oncology 2005;69:478-485.

19. Laakso M, Tanner M, Nilsson J, Wiklund T, Erikstein $\mathrm{B}$, Kellokumpu-Lehtinen $\mathrm{P}$, et al. Basoluminal carcinoma: A new biologically and prognostically distinct entity between basal and luminal breast cancer. Clin Cancer Res 2006;12:4185-91.

20. Gruvberger SK, Ringnér M, Edén $P$, Borg A, Fernö $\mathrm{M}$, Peterson $\mathrm{C}$, et al. Expression profiling to predict outcome in breast cancer: The influence of sample selection. Breast Cancer Res 2003;5: 23-6.

21. Sotiriou C, Neo SY, McShane LM, Korn EL, Long PM, Jazaeri A, et al. Breast cancer classification and prognosis based on gene expression profiles from a population-based study. Proc Natl Acad Sci USA 2003;100:10393-8.

22. Rakha EA, Putti TC, Abd El-Rehim DM, Paish C, Green AR, Powe DG, et al. Morphological and immunophenotypical analysis of breast carcinomas with basal and myoepithelial differentiation. J Pathol 2006;208:495-506.
23. Rouzier R, Perou CM, Symmans WF, Ibrahim $\mathrm{N}$, Cristofanilli $\mathrm{M}$, Anderson $\mathrm{K}$, et al. Breast cancer molecular subtypes respond differently to preoperative chemotherapy. Clin Cancer Res 2005;11:5678-85.

24. Sorlie T, Tibshirani R, Parker J, Hastie T, Marron JS, Nobel A, et al. Repeated observation of breast tumor subtypes in independent gene expression data sets. Proc Natl Acad Sci USA 2003;100:8418-23.

25. Birnbaum D, Bertucci F, Ginestier C, Tagett R, Jacquemier J, Charafe-Jauffret E. Basal and luminal breast cancers: Basic or luminous? Int J Oncol 2004;25:249-58.

26. Carey LA, Perou CM, Livasy CA, Dressler LG, Cowan D, Conway K, et al. Race, breast cancer subtypes, and survival in the Carolina Breast Cancer Study. JAMA 2006;295:2492-502.

27. Rodriguez-Pinilla SM, Sarrió D, Honrado E, Hardisson D, Calero F, Benitez J, et al. Prognostic significance of basal-like phenotype and fascin expression in node-negative invasive breast carcinomas. Clin Cancer Res 2006;12:1533-9.

Source of Support: Nil, Conflict of Interest: None declared.

\section{Author Help: Sending a revised article}

1) Include the referees' remarks and point to point clarification to those remarks at the beginning, in the revised article file itself. In addition, mark the changes as underlined or coloured text in the article. Please include in a single file
a. referees' comments
b. point to point clarifications on the comments
c. revised article with text highlighting the changes done

2) Include the original comments of the reviewers/editor with point to point reply, at the beginning of the article in the 'Article File'. To ensure that the reviewer can assess the revised paper in timely fashion, please reply to the comments of the referees/editors in the following manner:

- $\quad$ There is no data on follow -up of these patients.

Authors' Reply: The follow up of patients has been included in the results section [Page 3, para 2]

- $\quad$ Authors should highlight the relation of complication to duration of diabetes.

Authors' Reply: The complications as seen in our study group has been included in the results section [Page 4, Table] 